\title{
In Vitro Degradation of PHBV Scaffolds and nHA/PHBV Composite Scaffolds Containing Hydroxyapatite Nanoparticles for Bone Tissue Engineering
}

\author{
Naznin Sultana ${ }^{1,2}$ and Tareef Hayat Khan ${ }^{3}$ \\ ${ }^{1}$ Department of Mechanical Engineering, The University of Hong Kong, Pokfulam Road, Hong Kong \\ ${ }^{2}$ Medical Implant Technology Group (Mediteg), Department of Biomechanics and Biomedical Materials, FKBSK, \\ Universiti Teknologi Malaysia, Johor, 81310 Johor Bahru, Malaysia \\ ${ }^{3}$ Department of Architecture, FAB, Universiti Teknologi Malaysia, Johor, 81310 Johor Bahru, Malaysia
}

Correspondence should be addressed to Naznin Sultana, naznin@biomedical.utm.my

Received 11 January 2012; Revised 2 April 2012; Accepted 3 April 2012

Academic Editor: Krasimir Vasilev

Copyright (C) 2012 N. Sultana and T. H. Khan. This is an open access article distributed under the Creative Commons Attribution License, which permits unrestricted use, distribution, and reproduction in any medium, provided the original work is properly cited.

This paper investigated the long-term in vitro degradation properties of scaffolds based on biodegradable polymers and osteoconductive bioceramic/polymer composite materials for the application of bone tissue engineering. The threedimensional porous scaffolds were fabricated using emulsion-freezing/freeze-drying technique using poly(hydroxybutyrate-cohydroxyvalerate) (PHBV) which is a natural biodegradable and biocompatible polymer. Nanosized hydroxyapatite (nHA) particles were successfully incorporated into the PHBV scaffolds to render the scaffolds osteoconductive. The PHBV and nHA/PHBV scaffolds were systematically evaluated using various techniques in terms of mechanical strength, porosity, porous morphology, and in vitro degradation. $\mathrm{PHBV}$ and $\mathrm{nHA} / \mathrm{PHBV}$ scaffolds degraded over time in phosphate-buffered saline at $37^{\circ} \mathrm{C}$. $\mathrm{PHBV}$ polymer scaffolds exhibited slow molecular weight loss and weight loss in the in vitro physiological environment. Accelerated weight loss was observed in nHA incorporated PHBV composite scaffolds. An increasing trend of crystallinity was observed during the initial period of degradation time. The compressive properties decreased more than $40 \%$ after 5 -month in vitro degradation. Together with interconnected pores, high porosity, suitable mechanical properties, and slow degradation profile obtained from long-term degradation studies, the PHBV scaffolds and osteoconductive nHA/PHBV composite scaffolds showed promises for bone tissue engineering application.

\section{Introduction}

Polymer-based composite scaffolds seem to have great potential in bone tissue-engineering. By selecting proper materials and fabrication technologies, good-quality scaffolds with consistent properties can be possible to fabricate [1]. There are some basic requirements that have been widely accepted for designing polymer scaffolds which can significantly affect the cell seeding and growth both in vitro and in vivo [2, $3]$. The scaffolding materials should be biocompatible and biodegradable; the degradation products should be nontoxic and easily excreted by metabolic pathways. One of the key requirements is that the ideal scaffolding materials should be easy to fabricate into a desired shape, and they should have a controlled porous architecture to allow for cell infiltration, attachment, growth, tissue regeneration, and vascularization. The scaffolds should be mechanically strong to maintain their structural integrity during culture, and the ultimate mechanical properties of polymers at large deformations are important in selecting particular polymers for biomedical application.

Various biomaterials including biodegradable polymers, bioceramics, and biocomposites have been fabricated into scaffolds for the application of tissue regeneration. Poly(glycolic acid) (PGA), poly(lactic acid) (PLA), and their copolymers poly(lactic acid-co-glycolic acid) (PLGA) are 
the most frequently used materials which already have been fabricated into scaffolds for cell transplantation and tissue-engineering $[4,5]$. One of the materials of interest in tissue-engineering application is poly(hydroxybutyrate) (PHB) and its copolymer poly(hydroxybutyrate-cohydroxyvalerate) (PHBV) which can be derived by microorganisms via fermentation [6]. These polymers are composed of hydroxybutyrate (HB) units with between 0 and $24 \%$ of hydroxyvalerate (HV) units appearing randomly throughout the polymer chain [7]. The chemical structure of PHBV is given by

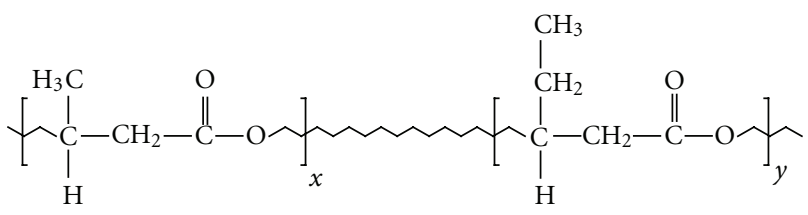

Poly(hydroxybutyrate-co-hydroxyvalerate) (PHBV)

This polymer is degradable and piezoelectric (and thus might promote bone growth in vivo) and possesses extremely good biocompatibility $[6,8]$. Together with highly biocompatibility, the copolymer PHBV has degradation times much longer than the other biocompatible polymers which will allow the scaffolds to maintain its mechanical integrity, until there is sufficient bone growth throughout the implant. PHBV copolymers have been found as minimal inflammatory in long-term studies of subcutaneous implants in mice and rats [9]. By considering these properties, PHBV copolymers may be a suitable candidate to support long-term bone regeneration [10]. The degradation products of $\mathrm{PHB}$ and PHBV polymers are the normal constituent of human blood, and hence they exert less inflammatory response to human body than other polymers $[6,11]$.

The uses of poly(hydroxyalkanoates) (PHA) could be extended considerably if a wider range of degradation profiles was available. The bone growth and healing can be stimulated theoretically as the polymer slowly degrades, by avoiding replacement operation. PHBV copolymers are also hydrolyzed in water with the normal universal acid-base catalysis for esters. At high $\mathrm{pH}$, the rate of degradation is quite rapid, but the hydrolysis proceeds very slowly in neutral buffer at body temperature. Moreover, the experiments suggested that the rate of degradation of $\mathrm{PHBV}$ in vivo is significantly faster than the in vitro hydrolysis rate at the same temperature and $\mathrm{pH}[12]$. Actually the nonspecific esterase and lysozyme enzymes secreted by the body's immune system catalyze the process. The variation of degradation rate of PHBV in vivo with the activity of the body's immune system thus is useful to explain some of the discrepancies in the literature of PHBV biodegradation. The range of biodeterioration of implanted films can be varied from very rapid to a modest but measurable resorption to virtually undetectable weight loss of fiber monofilament over an 18months period. It was also reported that PHBV and that cell/PHBV constructs have the ability to produce neocartilage in a heterotopic site although the degradation rates of
PHBV in different environments need more investigation [13].

It was reported that the in vivo and in vitro degradation of aliphatic polyesters was catalyzed by carboxyl end groups formed by chain cleavage, and the amorphous regions are preferentially degraded [14]. In general, the hydrolytic degradation of semicrystalline high molecular weight PLLA proceeds through random bulk hydrolysis in two distinct stages. The first stage is characterized by the preferential attack of the ester linkages in the more accessible amorphous regions, while the second stage is characterized by the attack of the less accessible crystalline regions [15]. It was reported that the cleavage of an ester bond of PLGA polymers yielded a carboxyl end group and a hydroxyl one, and thus formed carboxyl end groups were able to catalyze the hydrolysis of other ester bonds [14]. This phenomenon is called autocatalysis. Autocatalysis rate equation is applicable when the extent of reaction is slow or before the specimen experiences significant weight loss.

On the other hand, hydroxyapatite (HA) possesses osteoconductivity and is similar to the mineral component of natural bone. HA has been studied comprehensively. It is now widely used for bone tissue repair. Biodegradable composite scaffolds containing osteoconductive HA particles and biodegradable polymers are being explored [16, 17]. It was reported that by, incorporating nanosized HA particles in polymer scaffolds, the scaffolds should attain osteoconductive properties $[18,19]$. These scaffolds will be promising in bone tissue-engineering application.

In this paper, firstly, the study of fabrication and evaluation of PHBV and HA/PHBV composite scaffolds was reported. Then the results of in vitro degradation of pure PHBV scaffold, and HA-incorporated PHBV scaffolds were discussed from the materials perspective. However, report on long-term degradation of PHBV scaffolds is rarely available. This paper reports and compares the long-term systematic investigations of hydrolytic degradation characteristics of the scaffolds produced by PHBV polymers and osteoconductive nHA/PHBV composites. By undertaking long-term investigations, important information had been achieved in the understanding of the hydrolytic degradation characteristics of the scaffolds, particularly the slow degradation mechanism of PHBV scaffolds, and degradation induced morphological and mechanical changes of HA/PHBV composite scaffolds for the application of bone tissue-engineering.

\section{Materials of Methods}

2.1. Materials. Poly(hydroxybutyrate-co-hydroxyvalerate) (PHBV) as powder form was purchased from Tianan Biologic Material Ltd., Ningbo, China. PHBV with $2.9 \%$ of 3-hydroxyvalerate content had the molecular weight 310,000 and purity $98.8 \%$. These polymers were used without further purification. All the chemicals such as chloroform, acetic acid, and salts were analytical grade and were used as received. Water was ultra pure grade $(<18 \mathrm{~m} \Omega)$ supplied from a Mili-Q purification system. The HA nanoparticles used for composite scaffolds were produced in-house through a nanoemulsion process [20]. 
2.2. Fabrication and Characterization of Scaffolds. PHBV tissue-engineering scaffolds were fabricated using the emulsion freezing/freeze-drying technique described elsewhere $[17,18]$. Briefly, PHBV was weighed accurately into a centrifuge tube, and then an accurately measured amount of chloroform was added to the tube to make a solution with a desired concentration of $2.5 \%-12.5 \%(\mathrm{w} / \mathrm{v})$. To obtain a homogeneous polymer solution, the mixture was kept at $50^{\circ} \mathrm{C}$ in a water bath for five minutes and mixed thoroughly using a minishaker. The process was repeated for several times. After obtaining homogeneous solution, water phase was added. Homogenization at a fixed speed was performed in a homogenizer (Ultra-Turrax, T-25; IKA-WERKE). In order to fabricate scaffolds, $5 \mathrm{~mL}$ of PHBV emulsion was put into a glass vial (prewarmed to $50^{\circ} \mathrm{C}$ ). The glass vial containing the mixture was then rapidly transferred into a freezer at a preset temperature $\left(\right.$ at $-35^{\circ} \mathrm{C}$ ) to solidify the emulsion. The solidified emulsion was maintained at that temperature for overnight. The frozen emulsion was then transferred into a freeze-drying vessel (LABCONCO-Freeze dry system, USA) at a preset temperature of $-10^{\circ} \mathrm{C}$. The samples were freeze-dried for at least $46 \mathrm{hrs}$ to remove the solvent and water phase completely. The prepared scaffolds were placed in a vacuum desiccator at room temperature for storage and further removal of any residual solvent until characterization. The fabrication procedure of $\mathrm{nHA} / \mathrm{PHBV}$ scaffolds was similar as PHBV scaffolds. In order to fabricate 10\% HA-incorporated PHBV scaffold, 10\% HA (w/w) relative to the total amount of polymer was dispersed in the polymer emulsion ( $0.1 \mathrm{gHA}$ in $1 \mathrm{~g} \mathrm{PHBV})$ using the homogenizer. The other steps are similar as described above.

Using a scanning electron microscopy (SEM; Stereoscan $440, \mathrm{UK})$ at $12 \mathrm{kV}$, the pore structure and morphology of scaffolds were studied. The specimens were cut with a sharp razor blade after being frozen at $-35^{\circ} \mathrm{C}$ for one day. Then they were coated with gold using a sputter coater (BAL-Tec, SCD 005; Sputter Coater). The coating time was $200 \mathrm{sec}$. Pore sizes and pore size distributions were determined using SEM micrographs of scaffolds. At least 40 pores were measured from SEM micrographs, and the average pore size was calculated. HA nanoparticles were also coated with gold. By using a field emission-scanning electron microscope (FESEM, LEO 1530, Germany), the morphology of dried nHA was examined. Energy-dispersive X-ray spectrometry (EDX, INCA 300, UK) was performed in order to determine the presence and distribution of HA nanoparticles in composite scaffolds.

Using a differential scanning calorimetry (DSC, Pyris 6 , Perkin-Elmer, USA), the thermal properties of scaffolds were investigated. The degree of crystallinity, $X_{c}$, of scaffold samples was calculated with the use of DSC-melting curves of scaffolds. For composite scaffolds containing nHA, crystallinity is calculated using the equation given below [21]:

$$
X_{c}(\%)=\frac{\Delta H_{m} / \varphi_{\mathrm{PHBV}}}{\Delta H_{m} 100 \%} \times 100 \%,
$$

where $\Delta H_{m}$ and $\Delta H_{m 100 \%}$ are the calculated enthalpy of polymer scaffolds and the theoretical enthalpy of melting for $100 \%$ crystalline PHBV polymer $(114 \mathrm{~J} / \mathrm{g})$. The weight fraction of PHBV in the composite scaffolds is denoted by $\varphi_{\text {PHBV }}$. The skeletal density and the porosity of the scaffolds were measured according to liquid displacement method [22]. A scaffold sample of weight $W$ was immersed in a graduated cylinder containing a known volume $\left(V_{1}\right)$ of ethanol for $5 \mathrm{~min}$. Then a series of brief evacuationrepressurization cycles with the aid of a vacuum oven (DZF 6020 , China) was conducted to force the ethanol into the pores of the foam until no air bubbles were observed emerging from the foam. The total volume of ethanol and the ethanol-impregnated foam was then recorded as $V_{2}$. The ethanol-impregnated foam was removed from the cylinder, and the residual ethanol volume was recorded as $V_{3}$. The volume difference, $\left(V_{2}-V_{1}\right)$, was the volume of the polymeric foam, and the quantity $\left(V_{1}-V_{3}\right)$ was the volume of the ethanol held in the foam regarded as the void volume of the foam.

Thus the total volume of the scaffold is

$$
V=V_{2}-V_{3}
$$

Density of the foam is

$$
d=\frac{w}{V_{2}-V_{3}} \text {. }
$$

Porosity is

$$
\varepsilon=\frac{\left(V_{1}-V_{3}\right)}{\left(V_{2}-V_{3}\right)} .
$$

Compressive mechanical properties of PHBV and PHBVbased scaffolds were determined at room temperature using an Instron mechanical tester (Instron 5848, USA) with a $100 \mathrm{~N}$ load cell and at a crosshead speed of $0.5 \mathrm{~mm} / \mathrm{min}$. The compressive modulus was calculated from the initial linear region of stress-strain curves.

\subsection{In Vitro Degradation Study}

2.3.1. Experimental Setup. In order to study the aqueous degradation of PHBV and $10 \mathrm{wt} \% \mathrm{nHA} / \mathrm{PHBV}$ scaffolds, selected samples fabricated from $10 \%(\mathrm{w} / \mathrm{v})$ polymer solution were cut to the correct height $(1.5 \mathrm{~mm})$ and diameter $(10 \mathrm{~mm})$ with a sharp razor blade and weighed. Phosphatebuffered saline (PBS) was prepared by dissolving tablets of PBS (supplied by Zymed laboratories USA) with distilled water. The specimens were placed in sealable vials in $10 \mathrm{~mL}$ of PBS solution ( $\mathrm{pH}$ 7.4). The samples were pressed under PBS by applying vacuum. Air trapped in pores was removed by venting with the aid of a vacuum oven. PBS solution was replaced with new solution each week. At regular intervals, samples from buffer were removed, extensively rinsed with distilled water and dried under vacuum at $37^{\circ} \mathrm{C}$ and weighed. The experiment was performed for a period of 11 months and according to ASTM F 1635-04a standard test method [23]. The test method is intended to help assess the biodegradation rates (i.e., the mass loss rate) and changes in material or structural properties. 


\subsubsection{Property Assessment}

(1) Molecular Weight. Molecular weight was measured using a Zetasizer Nano series (Malvern 2000, Malvern Instruments Ltd., UK) which can perform molecular weight measurements using a process called static light scattering (SLS) which is a noninvasive technique used to characterise the molecules in a solution. Static light-scattering technique makes use of the time-averaged intensity of scattered light after the particles in a sample being illuminated by a light source such as laser. The initial molecular weight of the sample was measured. Samples were removed at each specified time period throughout the duration of the test, dried and tested for the molecular weight as above. The autocatalysis rate equation is given by

$$
\ln M_{n t}=-k t+\ln M_{n o},
$$

where, $M_{n t}$ is the molecular weight after in vitro degradation at time $t, M_{n o}$ is the initial molecular weight, and $k$ is the rate constant.

(2) Weight Loss. Three test samples were weighed prior to placement in the physiological solution. Upon completion of the specified time period, each sample was removed, gently rinsed with distilled water, and dried to a constant weight, and the weight loss was recorded. Weight loss during investigation was determined as

$$
\text { Weight loss }(\%)=\frac{\left(W_{i}-W_{f}\right)}{W_{i}} \times 100,
$$

where $W_{i}$ and $W_{f}$ are specimen weights before and after soaking in PBS.

(3) Water Uptake. In order to calculate water uptake, preweighed scaffolds specimens and thin films were removed periodically, washed with distilled water, blotted dry on filter paper in order to remove excess water, weighed, and returned to the PBS. The water uptake was calculated using the following equation:

$$
\text { Water uptake }(\%)=\frac{\left(W_{w}-W_{d}\right)}{W_{d}} \times 100,
$$

where $W_{d}$ and $W_{w}$ are specimen weights before and after soaking in PBS.

2.4. Characterization of Degraded Scaffolds. The compressive mechanical properties of the samples were determined prior to placement of the samples in the physiological solution (time zero). The samples were removed at each specified time period throughout the duration of the test and retest using the originally selected mechanical test methods and conditions. Crystallinities of as-fabricated and degraded scaffolds were also calculated as described earlier in Section 2.2. The morphologies of the as fabricated and degraded composite scaffolds were studied with a scanning electron microscopy (SEM; Stereoscan 440, Cambridge) at $12 \mathrm{kV}$ as described in Section 2.2.

\section{Results}

3.1. Properties of $P H B V$ and $n H A / P H B V$ Scaffolds. It was observed that, using freeze-drying technique, PHBV and nHA/PHBV scaffolds of high porosity (low skeletal density) could be fabricated from polymer solutions of different polymer concentrations. It was also noticed that the skeletal density increased while porosity decreased with the increasing polymer solution concentration. The porosity and skeletal density of scaffolds prepared from 5\% (w/v) PHBV solution were $88 \%$ and $0.1005\left(\mathrm{~g} / \mathrm{cm}^{3}\right)$, respectively, whereas the porosity and skeletal density of scaffolds prepared from $10 \%(\mathrm{w} / \mathrm{v})$ PHBV solution were $78 \%$ and $0.2244\left(\mathrm{~g} / \mathrm{cm}^{3}\right)$, respectively. The porosity decreased to $75 \%$, and skeletal density further increased to $0.2601\left(\mathrm{~g} / \mathrm{cm}^{3}\right)$ with the incorporation of $10 \mathrm{wt} \% \mathrm{HA}(\mathrm{w} / \mathrm{w})$ in $10 \%(\mathrm{w} / \mathrm{v}) \mathrm{PHBV}$ solution. The effects of polymer concentration on the final microstructure, pore sizes and porosity were also investigated (Figure 1). The scaffolds fabricated from 5\% (w/v) PHBV solution showed weak or low polymer interconnectivity Figures 1(a) and 1(b). On the other hand, the scaffolds produced from 10\% (w/v) PHBV solution exhibited highly anisotropic array of open microtubule with stronger pore interconnectivity (Figures $1(\mathrm{c})$ and $1(\mathrm{~d}))$. All the scaffolds exhibited open porous morphology. For the scaffolds fabricated from $10 \%(\mathrm{w} / \mathrm{v})$ solution had uniform pore structures which had the pore sizes ranging from 70 to $600 \mu \mathrm{m}$ with the average pore size of $297 \mu \mathrm{m}$. On the other hand, nHA/PHBV composite scaffolds had pore size range of $50-450 \mu \mathrm{m}$. The average pore size of nHA/PHBV composite scaffold was $210 \mu \mathrm{m}$.

Figure 2(a) shows the FE-SEM micrograph of nanosized HA. The HA nanoparticles had higher in vitro solubility under physiological conditions which suggested the higher bioresorbable nature [20]. The freeze-dried nHA powders used in this investigation consisted of tiny agglomerates of HA nanocrystallites. The particle size of the nHA powders was found to be in the range of $20-30 \mathrm{~nm}$ [20]. It was observed that at $10 \mathrm{wt} \% \mathrm{nHA}$ concentration, nHA particles were distributed homogeneously within the pore walls of the scaffolds. No significant agglomeration of HA nanoparticles occurred within the pore walls. Figure 2(b) shows the nHA/PHBV scaffold with the nHA content of $10 \mathrm{wt} \%$. The presence of nHA in polymer pore walls was confirmed by EDX spectrum and EDX analysis (Figure 2(c) and Table 1).

\subsection{Effect of Polymer Concentration on Compressive Mechan-} ical Properties. Figure 3(a) shows the typical compressive curves of the PHBV scaffolds made from the emulsion concentrations of $5 \%, 7.5 \%, 10 \%$, and $12.5 \%(\mathrm{w} / \mathrm{v})$. It can be observed from compressive curves that the compressive properties of the PHBV scaffolds increased with the increasing emulsion concentration. All the curves had three distinct regions consisting of initial linear region followed by a long plateau and then densification. The compressive modulus was calculated from initial linear region. The scaffolds of $7.5 \%(\mathrm{w} / \mathrm{v})$ polymer solution had the compressive modulus of $1.1 \pm 0.61 \mathrm{MPa}$ in the $2.5 \%-7.5 \%$ strain range whereas the scaffolds of $12.5 \%(\mathrm{w} / \mathrm{v})$ had the compressive modulus of $5.02 \pm 1.03 \mathrm{MPa}$ in the same strain range (Table 2 ). Figure 4 


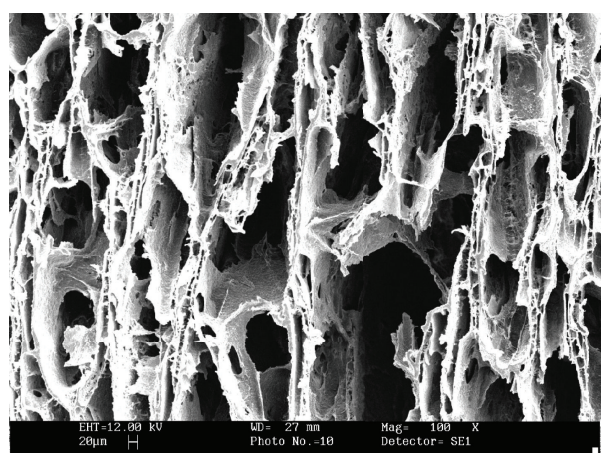

(a)

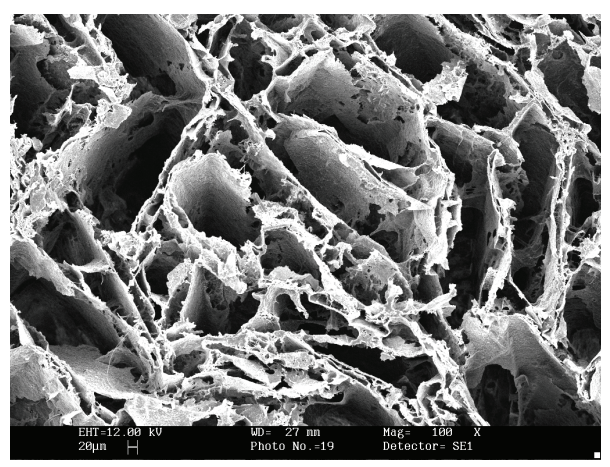

(c)

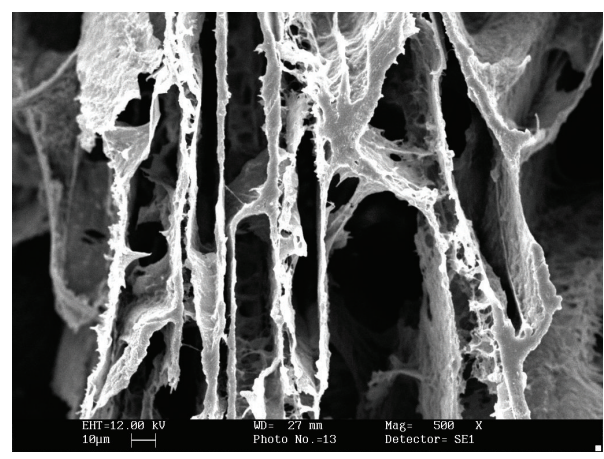

(b)

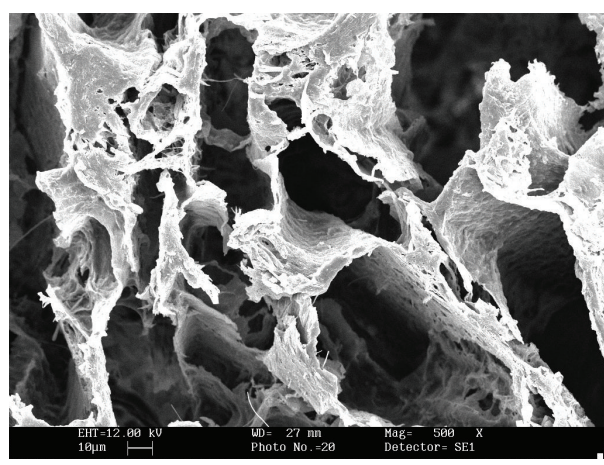

(d)

Figure 1: SEM micrographs of PHBV scaffolds fabricated by emulsion freezing/freeze-drying technique from (a) and (b) 5\% PHBV emulsion, and (c) and (d) 10\% PHBV emulsion.

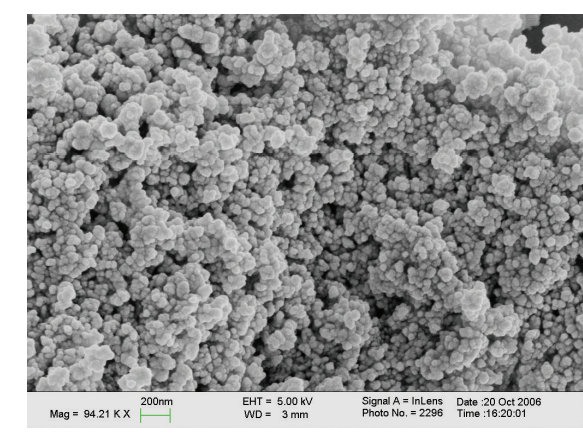

(a)

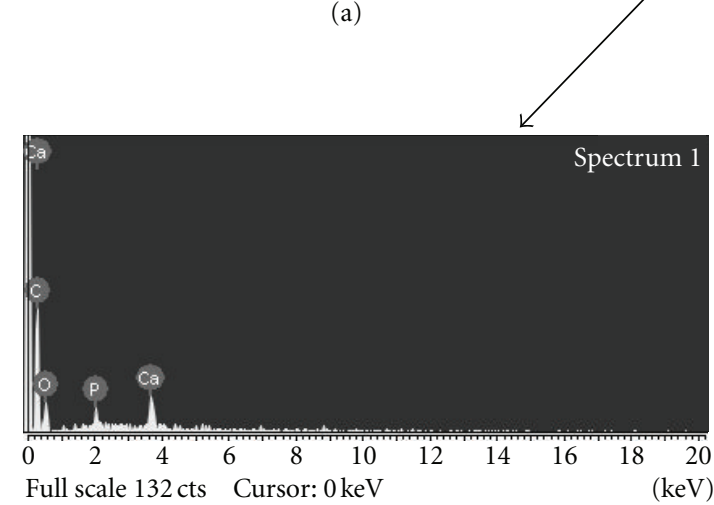

(c)

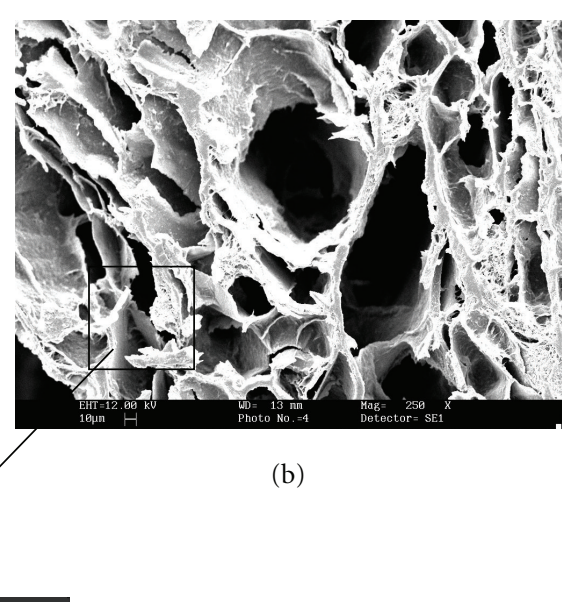

\footnotetext{
confirming the presence of HA nanoparticles in HA/PHBV composite scaffold.
}

FIGURE 2: (a) FE-SEM micrograph of nanosized HA particles; (b) HA/PHBV composite scaffold containing 10\% nHA; (c) an EDX spectrum 


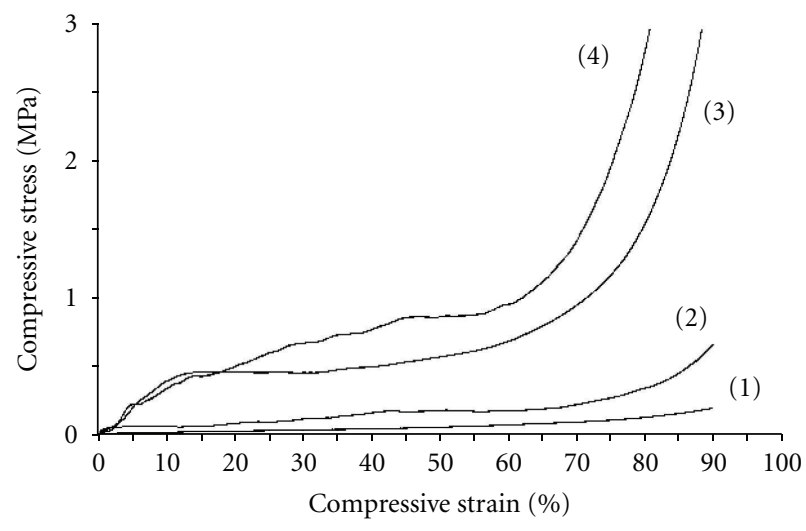

(a)

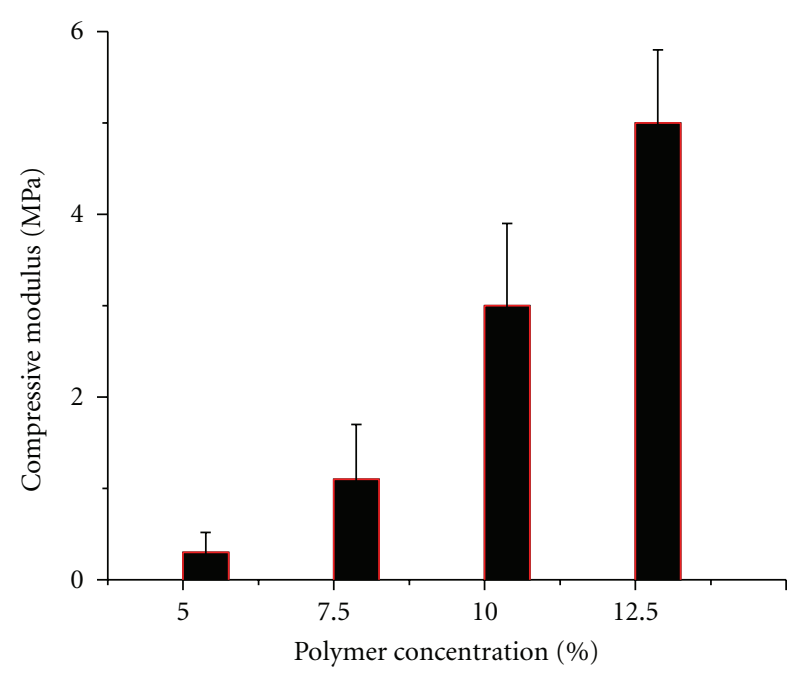

(b)

Figure 3: (a) Typical compressive curves of the PHBV scaffolds (1) 5\%, (2) 7.5\%, (3) 10\%, and (4) 12.5\% (w/v) polymer emulsions. (b) Compressive modulus of PHBV scaffolds with different polymer concentration.

TABLE 1: Elemental analysis of HA using EDX analysis of a HA/PHBV scaffold.

\begin{tabular}{lcc}
\hline \multirow{2}{*}{ Element } & \multicolumn{2}{c}{ Percentage of elements } \\
& Weight \% & Atomic \% \\
\hline $\mathrm{C}$ & 61.47 & 61.47 \\
$\mathrm{O}$ & 36.71 & 36.71 \\
$\mathrm{P}$ & 0.41 & 0.41 \\
$\mathrm{Ca}$ & 1.41 & 1.41 \\
\hline
\end{tabular}

TABle 2: Compressive modulus of PHBV scaffolds with different polymer concentration.

\begin{tabular}{lc}
\hline Polymer concentration & Compressive modulus $(\mathrm{MPa})$ \\
\hline $5 \%$ & $0.3 \pm 0.22$ \\
$7.5 \%$ & $1.1 \pm 0.61$ \\
$10 \%$ & $3.0 \pm 0.9$ \\
$12.5 \%$ & $5.0 \pm 0.8$ \\
\hline
\end{tabular}

shows the effects of scaffold porosity on the compressive modulus of the PHBV scaffolds. It was found that the compressive modulus decreased with the increasing porosity (Table 3).

3.3. Water Uptake. Figure 5 shows the comparison of the water uptake curves between PHBV polymer scaffold and $10 \%$ nHA in $\mathrm{PHBV}$ composite scaffolds at $37^{\circ} \mathrm{C}$. It was observed that the initial water uptake of HA-incorporated composite scaffold was much higher than that of polymer scaffold. Approximate equilibrium reached almost at the same immersion time for both of the scaffolds.

3.4. Molecular Weight Change of PHBV Scaffolds. Molecular weights of PHBV scaffolds immersed in the PBS solution

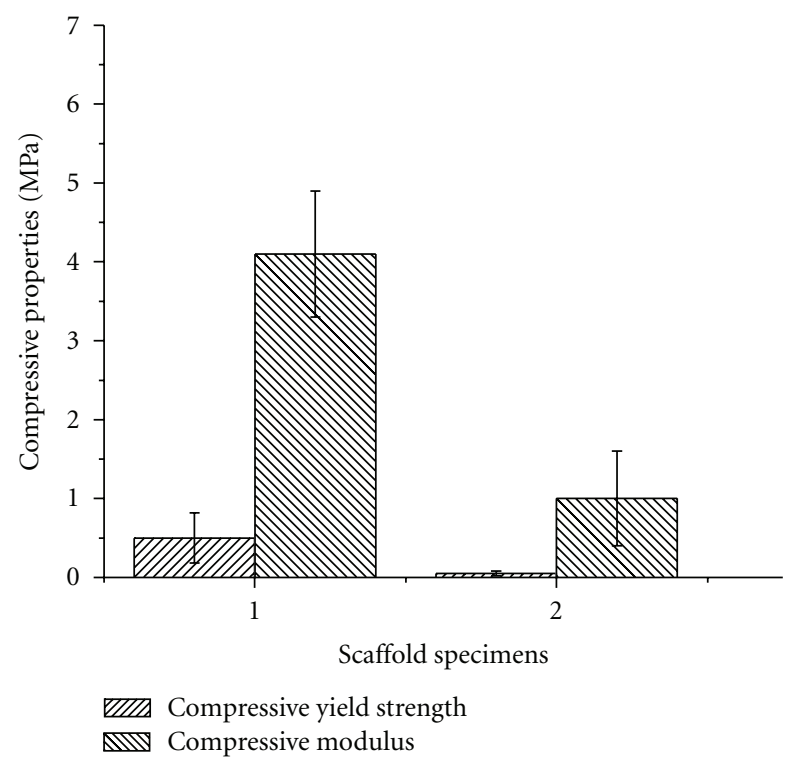

FIGURE 4: Effects of scaffold porosity on the compressive properties of the PHBV scaffolds: porosity (1) $77 \%$, (2) $83 \%$.

at $37^{\circ} \mathrm{C}$ were measured at different time points and the results were shown in Figure 6(a). It was observed that the molecular weight dropped significantly from 24 weeks to 44 weeks after in vitro degradation. The average molecular weight of as-fabricated PHBV scaffold was $229 \mathrm{KDa}$. After the scaffold immersion in PBS for 12 weeks, 14 weeks, and 44 weeks, it decreased to $212 \mathrm{KDa}, 194 \mathrm{KDa}$, and $121 \mathrm{KDa}$, respectively.

Figure 6(b) represents the plot of natural logarithm of molecular weights over degradation time for 44 weeks to fit the autocatalysis rate equation. As can be seen from Figure 6(b), the logarithm of molecular weight loss does not fall linearly over 44 weeks of time. But for the initial period 


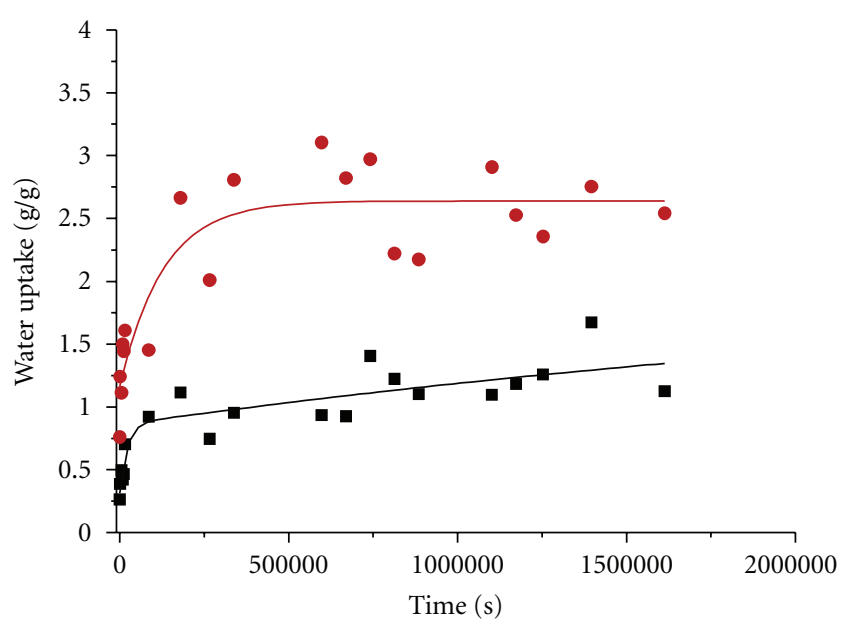

Figure 5: Comparison of water uptake between PHBV scaffolds ( and $10 \% \mathrm{HA}$ in PHBV composite scaffolds $(\bullet)$ at $37^{\circ} \mathrm{C}$.

TABlE 3: Compressive properties of the PHBV scaffolds with different porosity.

\begin{tabular}{lcc}
\hline Porosity & Compressive yield strength & Compressive modulus \\
\hline $77 \%$ & $0.50 \pm 0.31$ & $4.1 \pm 0.8$ \\
$83 \%$ & $0.052 \pm 0.03$ & $1.0 \pm 0.6$ \\
\hline
\end{tabular}

up to 24 weeks, the decrease in molecular weight followed the linear trend (Figure 6(b), minigraph). Here the autocatalysis reaction model can be applied. The slope of the straight line or the rate constant was found to be 0.0067 week $^{-1}$. It can be concluded from this result that the reaction rate is comparatively slower.

3.5. Crystallinity Change. It was observed that after 5week immersion in PBS, the crystallinity of pure PHBV scaffolds and 10\% nHA/PHBV scaffolds increased by about $8 \%$ (Figure 7 ), which was due to the degradation of the amorphous part of the polymers. This degradation was a result of hydrolytic scission of polymer chains in the more susceptible amorphous part. Moreover, the presence of nHA could accelerate the scaffold degradation by the dissolution of nano-HA in the scaffold matrices.

3.6. Weight Loss. Figure 8 (a) shows the effect of porosity on the weight loss of the scaffolds. It was observed that the scaffolds having higher porosity (88\%) exhibited accelerated weight loss than that of lower porosity (78\%). Figure 8 (b) displays the weight loss of the scaffold specimens of PHBV $(10 \% \mathrm{w} / \mathrm{v})$ and the same composition containing $10 \% \mathrm{HA}$ over 8 week period of time. After six weeks, the $10 \%$ nHA containing PHBV composite scaffold showed elevated weight loss (approx. 18\%) than polymer scaffolds $(<10 \%)$. Accelerated weight loss of nHA/PHBV composite scaffolds was due to dissolution of HA in the scaffolds matrix.

3.7. Changes of Compressive Mechanical Properties. Mechanical testing showed that compressive properties of $\mathrm{PHBV}$ scaffolds decreased considerably after 20-week immersion in PBS. Figure 9(a) shows the typical compressive stress-strain curves as fabricated, and Figure 9(b) shows the degraded PHBV scaffolds specimens fabricated from $10 \%$ (w/v) polymer emulsion. Compressive properties were determined from the initial linear part of the stress-strain curves. For each specimen compressive properties decreased more than $40 \%$ after in vitro degradation for 20 weeks at $37^{\circ} \mathrm{C}$ (Figure 9(c)).

3.8. Morphological and Microstructural Changes. Figures $10(\mathrm{a}), 10(\mathrm{~b})$, and 10 (c) show the morphology of PHBV scaffolds after in vitro degradation in $\mathrm{PBS}$ at $37^{\circ} \mathrm{C}$ for 4 weeks and 44 weeks. After 44 weeks, large pores were observed in the PHBV scaffolds. Figures $10(\mathrm{~d})$ and $10(\mathrm{e})$ show the morphological change of nHA/PHBV composite scaffolds containing $10 \%$ of HA after degradation tests. After 4 weeks in PBS, the pore distribution was found to be more irregular, and large pores were observed. After 44 weeks in PBS, major morphological changes were detected for composite scaffolds. After immersion in PBS, the pore walls were found to be collapsed in the composite scaffolds, which were not observed in the pure PHBV scaffolds.

\section{Discussion}

Polymer scaffolds prepared from the optimum parameters exhibited $>70 \%$ porosity and better handling properties. The optimum parameters were polymer concentration, $10 \%(\mathrm{w} / \mathrm{v})$; solvent to water phase ratio, $1: 1$ or the volume fraction of water phase $\phi=0.5$; amount of HA, $10 \%$ (w/w); homogenizer speed, 17,500 rpm; freezer temperature, $-35^{\circ} \mathrm{C}$. The pore size ranged from several microns to few hundred microns, and they changed with the concentration of the polymer. Figure 1 shows the morphology of PHBV scaffolds of different concentrations. Anisotropic pore morphology with elongated pores and internal ladder-like microstructures in the pores of scaffolds were observed. The characteristic pore morphology obtained from emulsion freezing/freeze-drying technique can be explained by the phase separation and heat transfer mechanisms during freezing and solvent removal rate by freeze-drying processes. Incorporation of nHA did not significantly change the pore microstructures as observed in Figure 2.

The compressive stress-strain curves had the three regions as shown in Figure 3. It was found that they exhibit linear elasticity at low stresses followed by a long collapse plateau and a regime of densification in which the stress rises steeply. It was reported that cell wall bending of the scaffolds controls the initial linear elasticity of the scaffolds [24]. Collapse of the cells is the cause of the plateau region. The final region of rapidly increasing stress occurs when the cells have almost completely collapsed. In this region, opposing cell walls touch, further strain compresses the solid itself [18, 24]. Compressive modulus is the initial slope of the stressstrain curve. As the relative density increases, the cell walls thicken and the pore space shrinks. Increasing the relative density of the foam increases the modulus, raises the plateau 


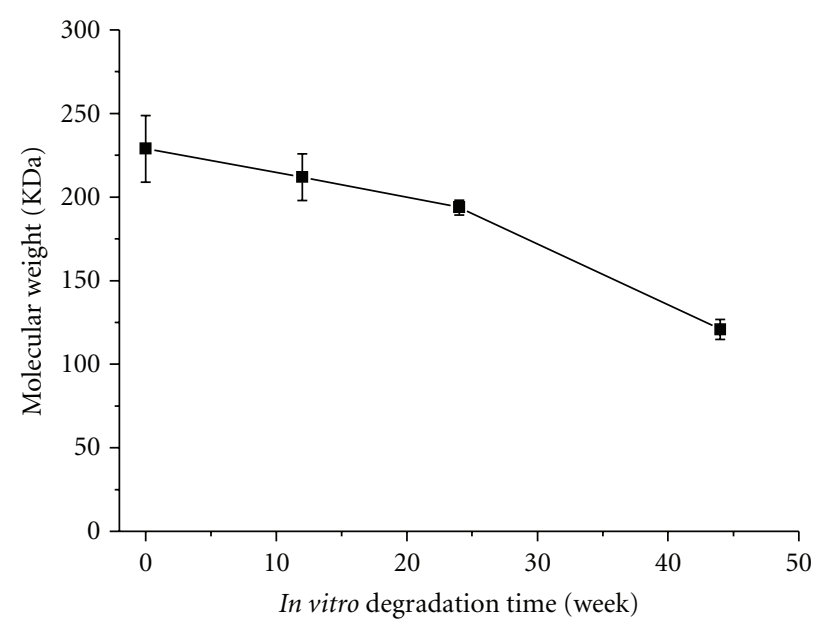

(a)

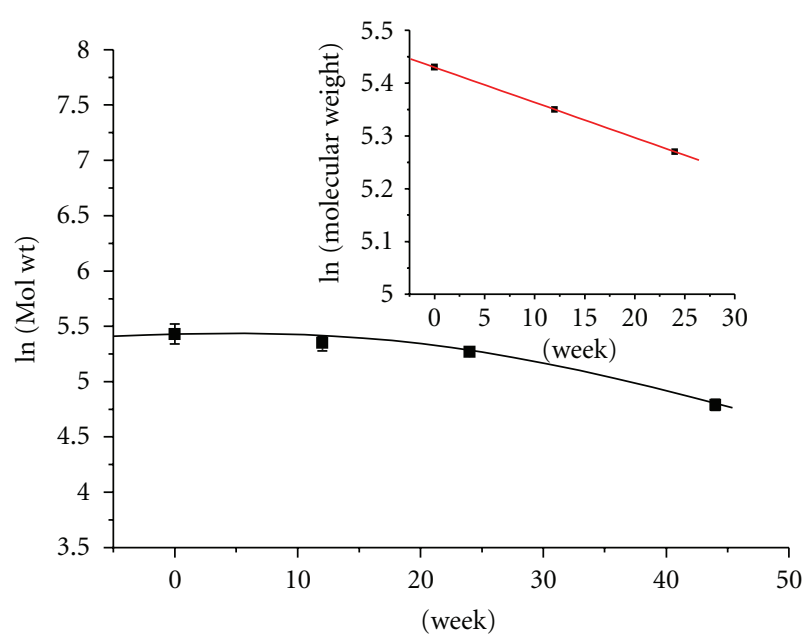

(b)

FIGURE 6: (a) Molecular weight (KDa) change with in vitro degradation time of PHBV scaffolds. (b) A plot of ln (molecular weight, KDa) with in vitro degradation of PHBV scaffolds for 44 weeks at $37^{\circ} \mathrm{C}$; (minigraph) plot of ln (molecular weight, $\mathrm{KDa}$ ) and degradation time (wk) at $37^{\circ} \mathrm{C}$ for 24 weeks.

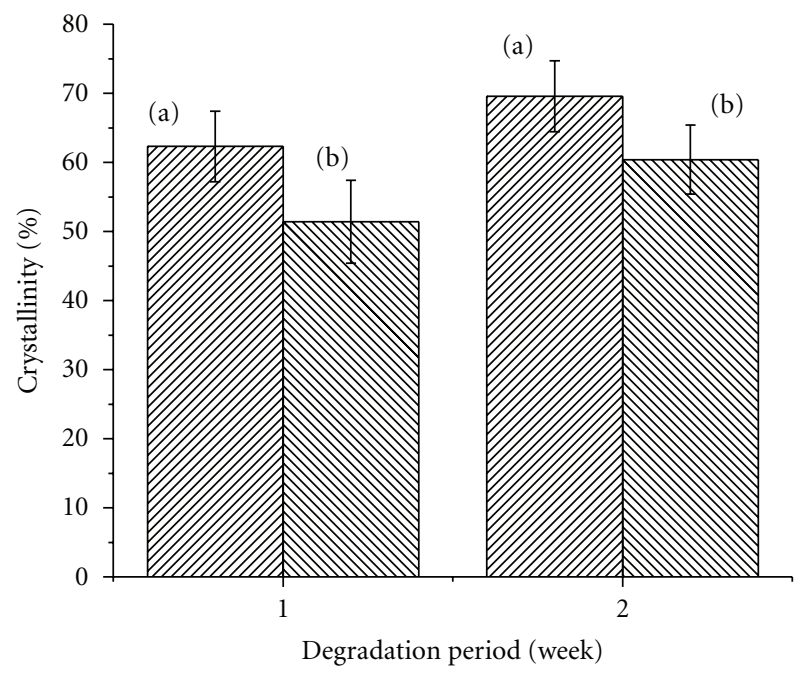

Figure 7: Crystallinity of (a) PHBV scaffolds and (b) $10 \%$ HA/PHBV scaffolds after (1) 0 week and (2) 5 weeks.

stress, and reduces the strain at which densification starts. For these reasons it was found that compressive modulus increases with increasing polymer/emulsion concentration or decreasing porosity (Figure 4).

The degradation mechanism and rate of biodegradable polymers can be affected by numerous factors. Among the factors which affect degradation are molecular weight, structure and content of comonomer unit, crystallinity, orientation, blending, porosity, $\mathrm{pH}$, temperature, and catalytic molecules or ions [25]. It was also reported that when catalytic molecules or substances such as enzymes and alkalis are present in the degradation media or environment, the degradation of polymer-based materials proceeds via a surface erosion mechanism [25]. In the surface erosion mechanism, catalytic molecules or ions act only on the surface of materials and will not diffuse into the material. As a result, the material is eroded from the surface while the core part of the material remains unchanged. On the other hand, the degradation of biodegradable polymers takes place via a bulk erosion mechanism in the absence of catalytic molecules or ions as in a phosphate-buffered solution. It was also reported that the hydrolytic degradation mechanisms depend on the thickness of biodegradable materials, and the critical thickness above which the degradation mechanism changes from bulk erosion to surface erosion depends on the molecular structure of biodegradable or hydrolysable polymers [26]. A significant weight loss can be observed at an early stage of degradation for a surface erosion mechanism. On the other hand, the weight loss occurs only at a late stage of degradation for a bulk erosion mechanism when a large decrease in molecular weight takes place and when water soluble oligomers and monomers are formed. In order to trace bulk erosion, molecular weight change is most effective. On the other hand, it is quite ineffective in the case of surface erosion [25].

Figure 6(b) shows that the autocatalytic rate equation is not valid over 11 months of period. On the other hand, Figure 6(b) (mini-graph) shows the plot between $\ln$ (Molecular weight) for 24 weeks of in vitro degradation time. During this time period, the rate equation for autocatalysis was valid as it followed first order with the regression coefficient $r^{2}$ value being 0.99 . The rate constant calculated from the slope was relatively small. It can demonstrate that the reaction proceeded slowly over this time period. It was reported that the reaction constant was 0.0584 week $^{-1}$ for PLGA [14]. This value was significantly greater than the value calculated for PHBV scaffolds in this study which was 0.0067 week $^{-1}$. It has been reported that surface area and thickness of film have little effect on the rate of 


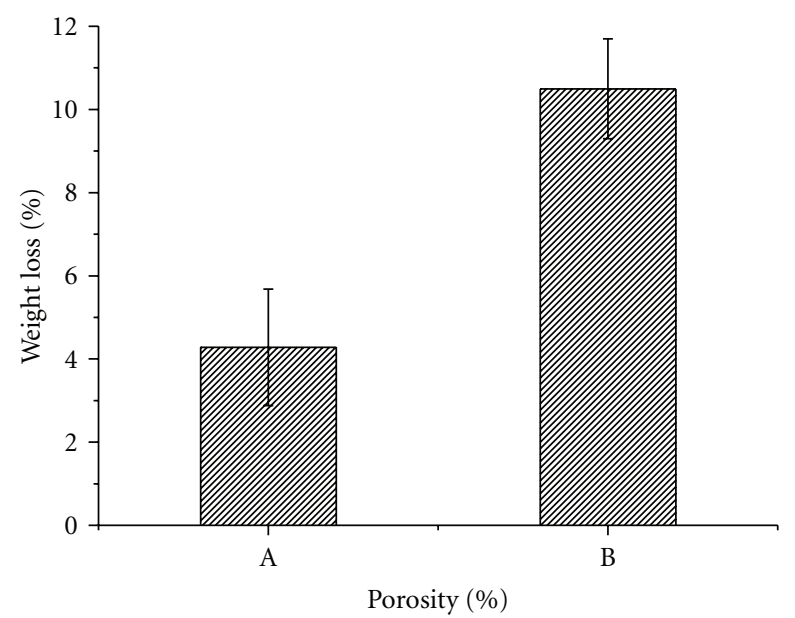

(a)

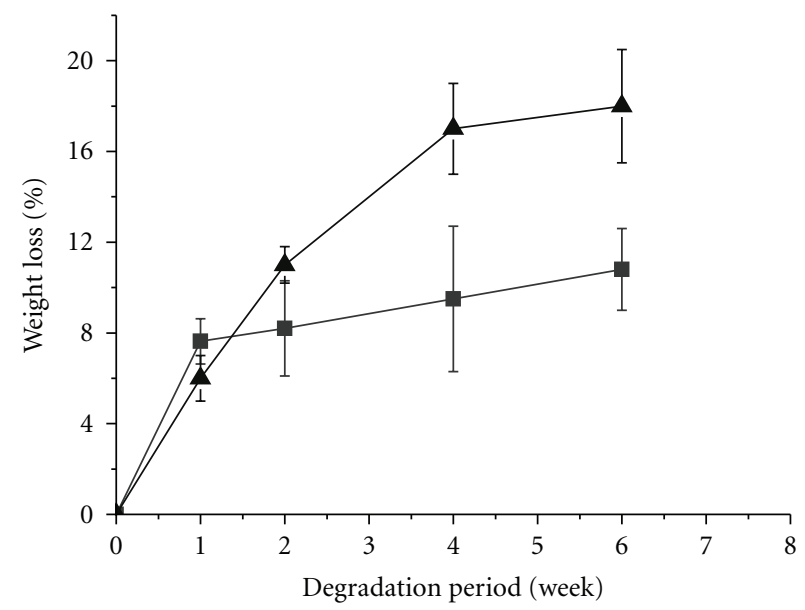

(b)

FIGURE 8: (a) Weight loss of PHBV scaffolds after in vitro degradation for 6 weeks: (A) 78\% porosity; (B) 88\% porosity. (b) Weight loss of PHBV scaffold (ם) and HA/PHBV scaffold with 10\% HA ( $\mathbf{(})$ after in vitro degradation for 6 weeks.

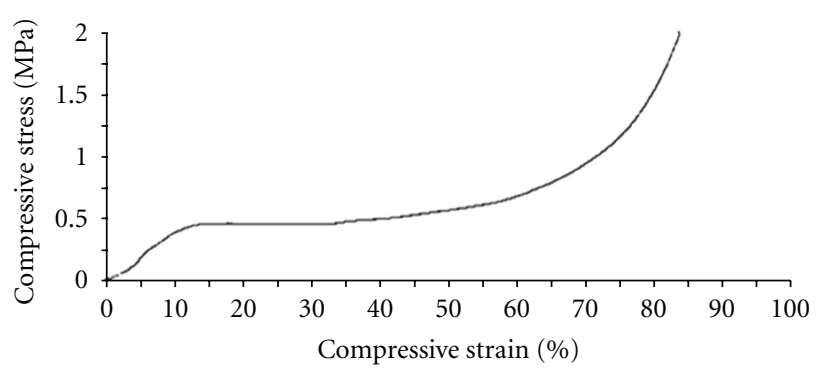

(a)

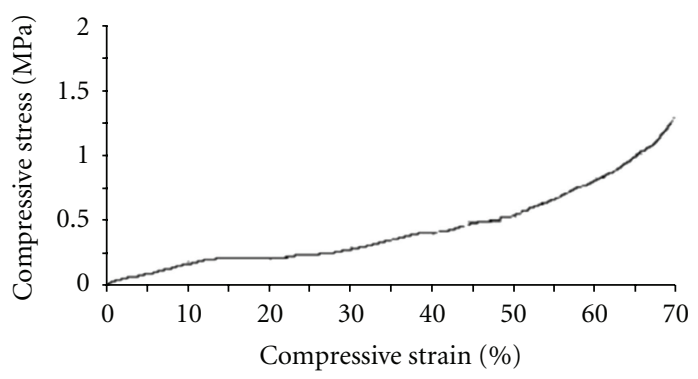

(b)

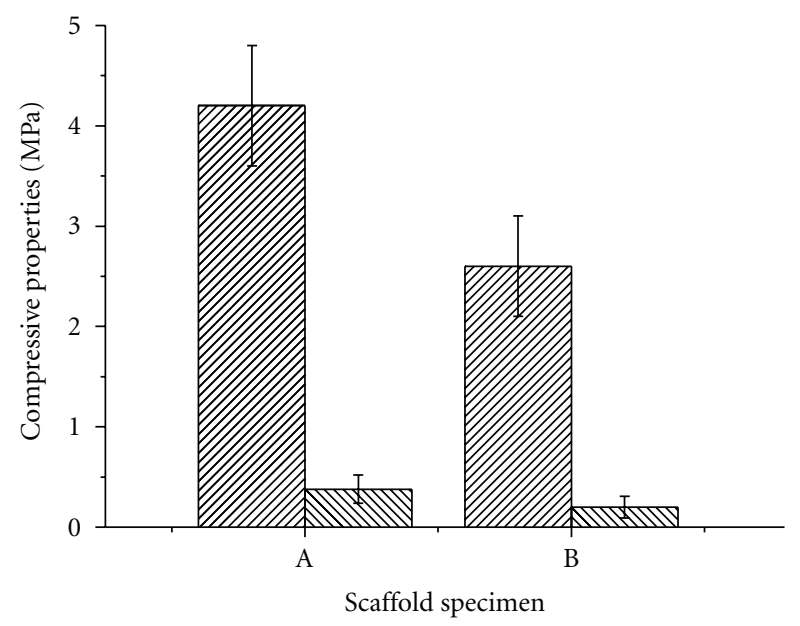

Compressive modulus

Compressive yield strength

(c)

FIGURE 9: Comparison of compressive stress-strain curves for (a) as-fabricated and (b) a degraded (20 weeks) PHBV scaffold specimens fabricated from 10\% (w/v) PHBV emulsion. (c) Compressive properties for an as-fabricated (A) and a degraded (20 weeks) (B) PHBV scaffold specimen. 


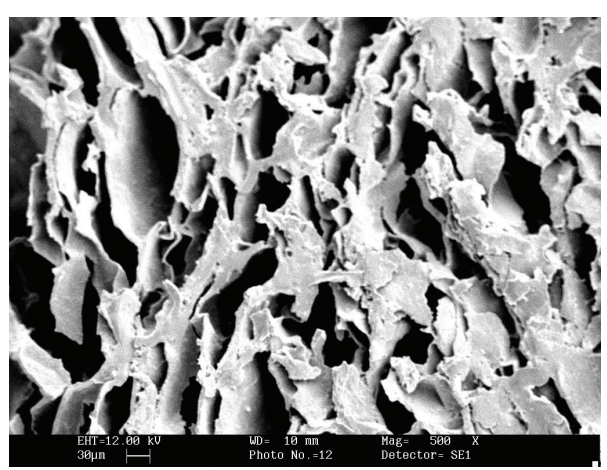

(a) In vitro degradation after 4 weeks

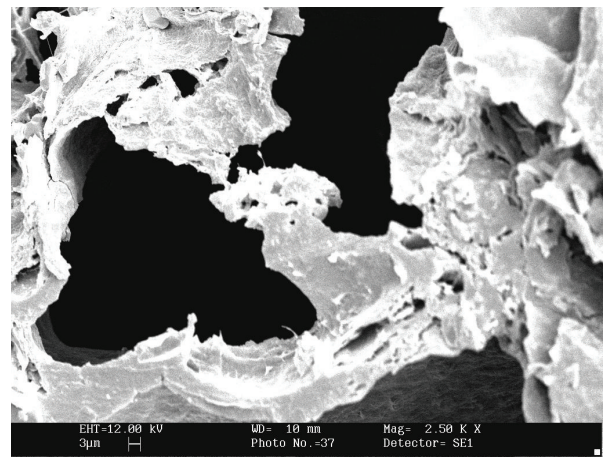

(c) In vitro degradation after 44 weeks (High magnification view)

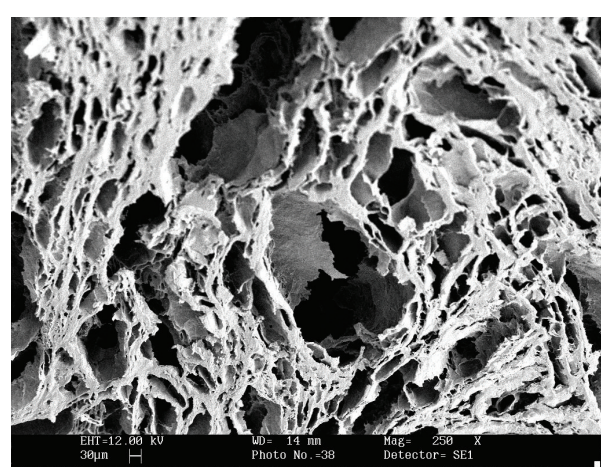

(b) in vitro degradation after 44 weeks

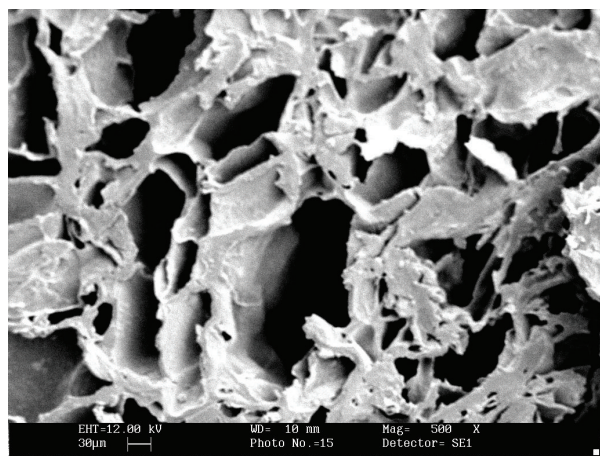

(d) In vitro degradation after 4 weeks

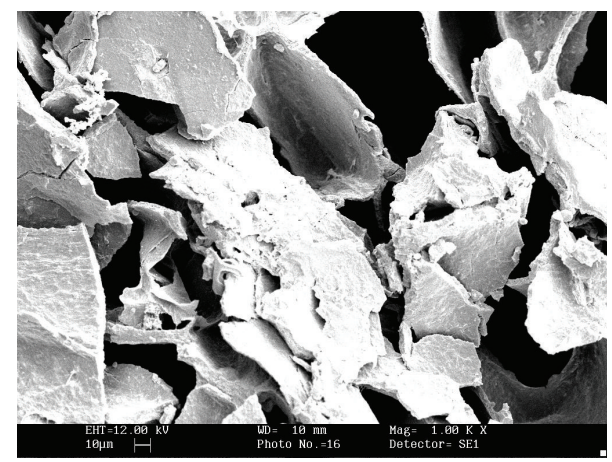

(e) In vitro degradation after 44 weeks

FIGURE 10: SEM micrographs of PHBV scaffolds after in vitro degradation in PBS at $37^{\circ}$ (a, b, and c); SEM micrographs of HA/PHBV composite scaffolds: after in vitro degradation for 4 weeks (d) and 24 weeks (e) in PBS at $37^{\circ}$.

biodegradation of $\mathrm{PHB}$ or PHBV. The degree of crystallinity is the major factor which controls the hydrolysis rate [27].

The weight loss can be caused by physical disintegration and fragmentation of scaffolds. The hydrolytic scission of polymer backbone in the scaffolds gradually produces shortchain monomer molecules that absorb more water molecules from the aqueous medium. Figure 8(a) deals with the scaffolds prepared from $5 \%(\mathrm{w} / \mathrm{v})$ and $10 \%$ emulsion concentration. As the scaffolds porosity increases with decreasing emulsion concentration, so it can be demonstrated that scaffolds weight loss increases with increasing porosity of the scaffolds. On the other hand, after certain period of time, the dissolution rate of nanosized HA may increase which can quicken the disintegration process (Figure $8(\mathrm{~b})$ ). For this reason, the weight loss of nHA-incorporated composite scaffolds was faster than PHBV scaffolds. Similar observations were obtained by several other research groups [8]. In vitro degradation experiment of phosphate glass-reinforced PHBbased degradable composites was studied in (PBS), and the results showed that the mass loss and mechanical property change could be closely correlated with the solubility rate of the reinforcing glass [8]. It was also demonstrated that the polymer was highly permeable.

Accelerated hydrolysis at elevated temperatures (55 and $70^{\circ} \mathrm{C}$ ) was also investigated [28], and it was found that a homogeneous process was involved which had two stages. Above $60^{\circ} \mathrm{C}$, there was no induction period, and the initial random scission of the ester groups occurred throughout 
the polymer (amorphous and crystalline regions) which directed to a decrease in molecular weight but little change in the polydispersity and almost no bulk mass loss. As the scaffolds are intended to apply for biomedical application in the current study, experiments were conducted mainly on physiological temperature and condition.

It was established that the strength and stiffness of the materials reduced on in vitro environment exposure in $\mathrm{PBS}$ at $37^{\circ} \mathrm{C}$ for periods up to 4 months (Figure 9). The degradation rate was a function of composition and processing conditions, and the materials did not show any conclusive evidence of extensive structural breakdown in vitro during the study period. Major morphological changes were observed in composite scaffolds after the study period (Figures 10(d) and 10(e)). Fabrication, characterization, and in vitro degradation of composite scaffolds based on PHBV and bioactive glass (BG) were performed by another research group [29]. These results suggested that incorporation of BG into PHBV could improve the hydrophilicity of the composites, and the enhancement was dependent on the BG content. In the current study, the degradation assessment of the scaffolds was performed in PBS at $37^{\circ} \mathrm{C}$, and the measurement of molecular weight loss of the PHBV scaffolds showed that the degradation rate is slow. The weight loss, crystallinity, and compressive mechanical properties of PHBV scaffolds were also monitored and compared with that of $10 \% \mathrm{nHA} / \mathrm{PHBV}$ composite scaffolds. The nanosized HA particles in the composite scaffolds accelerated the weight loss of the composite scaffolds due to dissolution of nHA particles and capillary water uptake through the interfaces of nHA particles. This process can increase the hydrolytic attack of the polymer matrix. The increasing trend of crystallinity of PHBV scaffolds and nHA/PHBV scaffolds was due to the degradation of the amorphous part of the polymers. This degradation was a result of hydrolytic scission of polymer chains in the more susceptible amorphous part of the scaffold matrix. The decrease in compressive properties can emphasize the fact that degradation proceeded with random cleavage of polymer chain backbone. The in vitro biological evaluation study was reported separately [19].

\section{Conclusions}

The scaffolds fabricated using emulsion freezing/freezedrying technique exhibited the combined properties of high porosity, relatively low density, and optimum pore size distribution with good compressive mechanical properties. PHBV and nHA/PHBV scaffolds underwent hydrolytic degradation at $37^{\circ} \mathrm{C}$. Mass losses, molecular weight loss of $\mathrm{PHBV}$, decrease in compressive mechanical properties, increase in polymer crystallinity, and loss of structural integrity were observed during degradation. It was observed that autocatalytic rate equation can be applied for first 24 weeks of in vitro degradation. Successful fabrication of polymer and osteoconductive composite scaffolds with controlled porosity, pore size distribution together with slow and controlled degradation properties suggests great promise for the bone tissue-engineering applications.

\section{Acknowledgments}

N. Sultana thanks The University of Hong Kong (HKU) for providing her with a research studentship. This paper is a part of PhD thesis submitted to the University of Hong Kong by N. Sultana. Supervision of Professor Min Wang (HKU) is acknowledged. Assistance provided by Dr. Zhou Wenyou for the synthesis of HA is also acknowledged. This work was supported by a GRF grant (HKU 7182/05E) from the Research Grants Council of Hong Kong. N. Sultana and T. H. Khan also acknowledge the financial support provided by UTM research grant GUP Tier 2 (vote: Q.J130000.7136.03J35), (vote: Q.130000.7136.03J33), Ministry of Higher Education (MOHE) and RMC.

\section{References}

[1] L. G. Griffith and G. Naughton, "Tissue engineering-current challenges and expanding opportunities," Science, vol. 295, no. 5557, pp. 1009-1014, 2002.

[2] B. D. Ratner, Biomaterials Science: An Introduction to Materials in Medicine, Elsevier Academic Press, London, UK, 2nd edition, 2004.

[3] P. X. Ma, "Scaffolds for tissue fabrication," Materials Today, vol. 7, no. 5, pp. 30-40, 2004.

[4] K. U. Lewandrowski, Tissue Engineering and Biodegradable Equivalents: Scientific and Clinical Applications, Dekker, New York, NY, USA, 2002.

[5] J. B. Park and J. D. Bronzino, Eds., Biomaterials: Principles and Applications, CRC Press, Boca Raton, Fla, USA, 2003.

[6] P.A. Holmes, Developments in Crystalline Polymers-2, Edited by D. C. Basset, Elsevier Applied Science, London, UK, 1987.

[7] W. D. Luzier, "Materials derived from biomass/biodegradable materials," Proceedings of the National Academy of Sciences of the United States of America, vol. 89, no. 3, pp. 839-842, 1992.

[8] J. C. Knowles, G. W. Hastings, H. Ohta, S. Niwa, and N. Boeree, "Development of a degradable composite for orthopaedic use: in vivo biomechanical and histological evaluation of two bioactive degradable composites based on the polyhydroxybutyrate polymer," Biomaterials, vol. 13, no. 8, pp. 491-496, 1992.

[9] S. Gogolewski, M. Jovanovic, S. M. Perren, J. G. Dillon, and M. K. Hughes, "Tissue response and in vivo degradation of selected polyhydroxyacids: Polylactides (PLA), poly(3hydroxybutyrate) (PHB), and poly(3- hydroxybutyrate-co-3hydroxyvalerate) (PHB/VA)," Journal of Biomedical Materials Research, vol. 27, no. 9, pp. 1135-1148, 1993.

[10] A. Kumarasuriyar, R. A. Jackson, L. Grøndahl, M. Trau, V. Nurcombe, and S. M. Cool, "Poly( $\beta$-hydroxybutyrateco- $\beta$-hydroxyvalerate) supports in vitro osteogenesis," Tissue Engineering, vol. 11, no. 7-8, pp. 1281-1295, 2005.

[11] C. Bastioli, Handbook of Biodegradable Polymers, Rapra Technology, Shrewsbury, UK, 2005.

[12] S. F. Williums and D. P. Martin, "Applications of PHAs in medicine and pharmacy," in Biopolymers: Polyesters III, Y. Doi and A. Steinbüchel, Eds., p. 91, Wiley-VCH, Weinheim, Germany, 2002.

[13] J. Liu, B. Zhao, Y. Zhang, Y. Lin, P. Hu, and C. Ye, "PHBV and predifferentiated human adipose-derived stem cells for cartilage tissue engineering," Journal of Biomedical Materials Research. Part A, vol. 94, no. 2, pp. 603-610, 2010. 
[14] S. Li, "Degradation of biodegradable aliphatic polyesters," in Scaffolding in Tissue Engineering, P. X. Ma and J. Elisseeff, Eds., Taylor \& Francis, 2006.

[15] N. A. Weir, F. J. Buchanan, J. F. Orr, and G. R. Dickson, "Degradation of poly-L-lactide. Part 1: in vitro and in vivo physiological temperature degradation," Proceedings of the Institution of Mechanical Engineers, Part H, vol. 218, no. 5, pp. 307-319, 2004.

[16] H. W. Tong, M. Wang, Z. Y. Li, and W. W. Lu, "Electrospinning, characterization and in vitro biological evaluation of nanocomposite fibers containing carbonated hydroxyapatite nanoparticles," Biomedical Materials, vol. 5, no. 5, Article ID 054111, 2010.

[17] N. Sultana and M. Wang, "PHBV/PLLA-based composite scaffolds containing nano-sized hydroxyapatite particles for bone tissue engineering," Journal of Experimental Nanoscience, vol. 3, no. 2, pp. 121-132, 2008.

[18] N. Sultana and M. Wang, "Fabrication of HA/PHBV composite scaffolds through the emulsion freezing/freeze-drying process and characterisation of the scaffolds," Journal of Materials Science: Materials in Medicine, vol. 19, no. 7, pp. 2555-2561, 2008.

[19] N. Sultana and M. Wang, "PHBV/PLLA-based composite scaffolds fabricated using an emulsion freezing/freeze-drying technique for bone tissue engineering: surface modification and in vitro biological evaluation," Biofabrication, vol. 4, no. 1, Article ID 015003, 2012.

[20] W. Y. Zhou, M. Wang, W. L. Cheung, B. C. Guo, and D. M. Jia, "Synthesis of carbonated hydroxyapatite nanospheres through nanoemulsion," Journal of Materials Science: Materials in Medicine, vol. 19, no. 1, pp. 103-110, 2008.

[21] J. F. Zhang and X. Sun, "Mechanical properties and crystallization behavior of poly(lactic acid) blended with dendritic hyperbranched polymer," Polymer International, vol. 53, no. 6, pp. 716-722, 2004

[22] Y. Y. Hsu, J. D. Gresser, D. J. Trantolo, C. M. Lyons, P. R. J. Gangadharam, and D. L. Wise, "Effect of polymer foam morphology and density on kinetics of in vitro controlled release of isoniazid from compressed foam matrices," Journal of Biomedical Materials Research, vol. 35, no. 1, pp. 107-116, 1997.

[23] ASTM standard F 1635-04a, "Standard test method for in vitro degradation testing of hydrolytically degradable polymer resins and fabricated forms for surgical implants," ASTM International, West Conshohocken, Pa, USA, 2004.

[24] L. J. Gibson and M. F. Ashby, Cellular Solids : Structure and Properties, Cambridge Solid State Science Series, Cambridge University Press, Cambridge, UK, 2nd edition, 1997.

[25] H. Tsuji, Degradation of Poly (lactide)—Based Biodegradable Materials, Nova Science, New York, NU, USA, 2008.

[26] F. V. Burkersroda, L. Schedl, and A. Göpferich, "Why degradable polymers undergo surface erosion or bulk erosion," Biomaterials, vol. 23, no. 21, pp. 4221-4231, 2002.

[27] W. Amass, A. Amass, and B. Tighe, "A review of biodegradable polymers: uses, current developments in the synthesis and characterization of biodegradable polyesters, blends of biodegradable polymers and recent advances in biodegradation studies," Polymer International, vol. 47, no. 2, pp. 89-144, 1998.

[28] A. C. Albertsson, Degradable Aliphatic Polyesters. Advances in Polymer Science, Springer, Berlin, Germany, 2002.
[29] H. Li, R. Du, and J. Chang, "Fabrication, characterization, and in vitro degradation of composite scaffolds based on PHBV and bioactive glass," Journal of Biomaterials Applications, vol. 20, no. 2, pp. 137-155, 2005. 

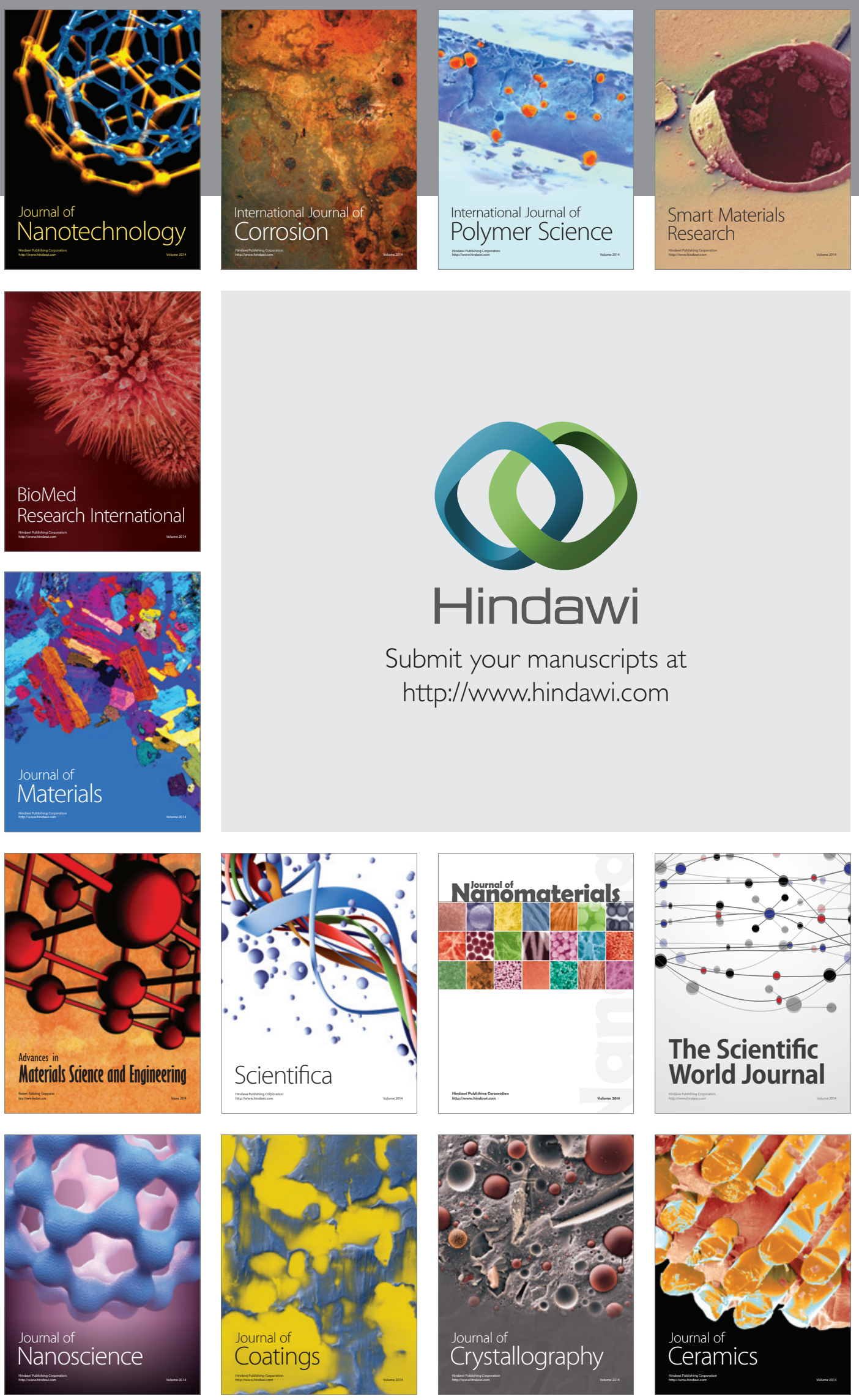

The Scientific World Journal

Submit your manuscripts at

http://www.hindawi.com

\section{World Journal}

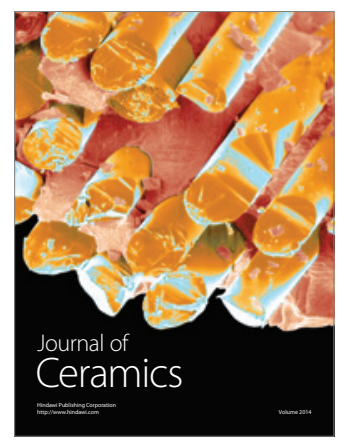

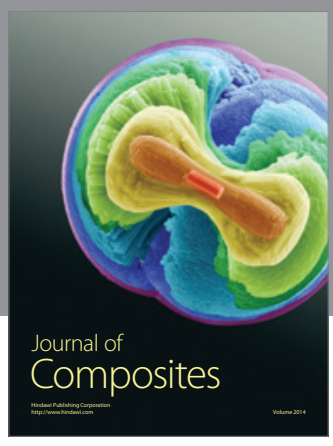
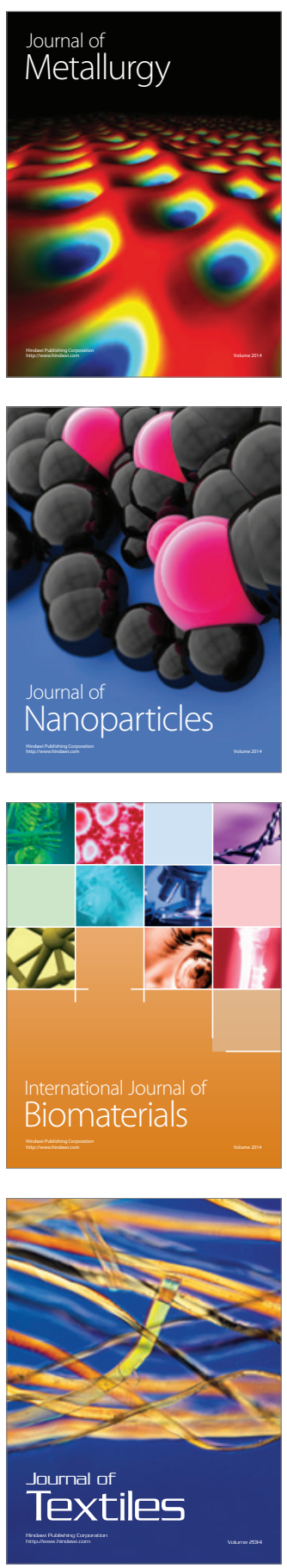